Journal was edited, for the first time, by two professors of psychiatry (A. H. Crisp and J. P. Watson) and, as a result, it became more diversified in content and included increasing proportions of empirical studies. This gradual shift in emphasis won some readers but lost others. Clinical psychologists became more interested by dynamic psychotherapists lost interest. This might have opened the way, in 1984, for the introduction of the new British Journal of Psychotherapy. The 1980s saw a steady growth in interest in health psychology and, because the BPS had no specialist journal for this area the term "medical" in the Journal's title took on a new significance. More and more health psychology papers were accepted until by 1990 , they had taken over almost half the Journal's contents. A recent policy change by the BPS Journals Committee has now diverted most of them to a special section of the British Journal of Clinical Psychology. Already the Journal is assuming more of its former character.

Present editorial policy needs to take account of the fact that the professions of psychiatry and clinical psychology have become progressively less clearly distinguishable from each other. Clinical psychologists have made important theoretical contributions to mental science and introduced therapeutic procedures which have been taken up by psychiatrists. Their activities have extended into areas which until recently were the exclusive domain of psychiatrists. It is acknowledged that the influence of psychoanalysis has diminished with the proliferation of new theories and methods of psychological intervention, and while remaining true to its psychoanalytic roots, the Journal now needs to expand outwards to incorpor- ate all possible theoretical models and psychotherapeutic approaches. We would like to see the Journal's contents divided equally between psychiatric and psychological contributions and collaborative studies between psychiatrists and psychologists are presently encouraged. There are roughly equal numbers of psychiatrists and psychologists on the editorial team. An ideal arrangement would be for its management to be shared between the BPS and the College, though we appreciate that this is an unrealisable dream.

It has often been considered that the British Journal of Psychiatry represented the more organic end of the psychiatric spectrum and the British Journal of Medical Psychology the more psychodynamic end. We are aware that in recent years the British Journal of Psychiatry has come to adopt a more liberal policy and to encourage submissions across the entire spectrum. This we welcome, but we would maintain that the British Journal of Medical Psychology continues to be a specialist journal in the fields of interpersonal relationships, psychodynamics and psychotherapy and we would wish to encourage more psychiatrists with interests in these fields to read, support and contribute to it. We remain conscious of the Journal's historic function of providing a meeting point for the disciplines of psychology and psychiatry, particularly in the interpersonal domain, and recognise that, in the coming years, as the two professions draw closer together, this function will become increasingly relevant. We respect the fact that psychiatry covers a broad spectrum and, while the Journal's interests lie predominantly at the relationships end, we recognise the need for the entire range of psychiatric journals.

\title{
A prize for the editor
}

Five years ago the Bruce Burns Memorial Trust was launched to commemorate the work of Consultant Psychiatrist Dr Burns, and in particular, his involvement with the promotion of mental health. At the time of his death in 1986 Dr Burns was Chairman of the West Midlands Working Party on Mental Health Promotion. The Memorial Trust's aim is to improve the mental health of people in the Region - especially former patients - by increasing awareness of the value of mental health promotion among both professional and lay people. One fruitful area has been the development of links between the arts and mental health services, encouraging the provision of more cultural opportunities for people who have had mental health problems. Projects include supporting theatre groups dealing with health issues and health education, and having visiting 'resident' artists working in hospitals.

The Trust's work falls into several different areas. It offers financial support to individuals or groups wishing to study topics or undertake research related to the promotion of mental health. There is also an annual guest lecture (the first one in 1988 was given by Professor Andrew Sims on 'How to Stay Sane'), and an award for the best published article aimed at the general public. This year's winner was Bulletin editor, Greg Wilkinson, whose booklet Depression has been highly praised. Dr Wilkinson was given the prize by the Chairman of the Trust, Dr A. C. White, at the 1991 annual lecture 'Schizophrenia-the beginning and the end' delivered in Birmingham in October by Professor Robin Murray. 